\title{
Effects of Dysglycemia and Dyslipidemia on the Severity of Airflow Limitation in Patients with Chronic Obstructive Pulmonary Disease
}

L. LI*

Department of Respiratory Medicine, Baise People's Hospital, No 8, Chengxiang road, Guangxi 533000, China

Li et al.: Effects of dysglycemia and dyslipidemia on airflow limitation in patients with COPD

To study the effect of dysglycemia and dyslipidemia on the severity of airflow limitation in patients with chronic obstructive pulmonary disease, 292 patients with moderate and higher chronic obstructive pulmonary disease were collected from a hospital during the period November 2018 to November 2019. They were divided into the dysglycemia group $(n=119)$ and the non-dysglycemia group $(n=173)$, the hyperlipemia group $(n=106)$ and non-hyperlipemia group $(n=186)$. Lung function index including the forced expiratory volume in $1 \mathrm{~s}$, forced expiratory volume 1/forced vital capacity and the forced expiratory volume in $1 \mathrm{~s}$ predicted. The blood gas indices including the partial pressure of carbon dioxide in the artery, the arterial partial pressure of oxygen and dyspnea index modified by the British Medical Research Council. Results indicated that the values of forced expiratory volume, forced expiratory volume $1 \%$ predicted and forced expiratory volume 1 /forced vital capacity in chronic obstructive pulmonary disease patients with dysglycemia were significantly higher than those in patients without dysglycemia $(p<0.05)$. The values of forced expiratory volume 1 , forced expiratory volume $1 \%$ predicted, and forced expiratory volume 1 /forced vital capacity in patients with chronic obstructive pulmonary disease in hyperlipidemia group were significantly higher than those in the non-hyperlipidemia group $(\mathbf{p}<0.05)$. Compared to the group without dysglycemia, the value of partial pressure of carbon dioxide in the artery in chronic obstructive pulmonary disease patients with dysglycemia was significantly higher $(p<0.05)$, and the partial pressure of oxygen value was significantly lower $(p<0.05)$. The partial pressure of carbon dioxide in the artery value of chronic obstructive pulmonary disease patients with hyperlipidemia was significantly higher than that of the non-hyperlipidemia group $(p<0.05)$ and the partial pressure of oxygen value was significantly lower $(p<0.05)$. The dispnea index modified by the British medical research council of the dysglycemia group was significantly higher than that of the non-dysglycemia group $(p<0.05)$ and the modified dispnea index of the hyperlipidemia group was significantly higher than that of the nonhyperlipidemia group $(\mathbf{p}<\mathbf{0 . 0 5})$. Diabetes or hyperlipidemia will aggravate the severity of airflow limitation in patients with chronic obstructive pulmonary disease and dysglycemia and/or dyslipidemia were the risk factors of chronic obstructive pulmonary disease.

Key words: Blood glucose, blood lipid, chronic obstructive pneumonia, the severity of airflow limitation

Chronic obstructive pulmonary disease (COPD) was a common respiratory disease, the clinical manifestations of patients were not completely reversible chronic airflow obstruction, dyspnea, systemic inflammatory reaction, which seriously affect the patient's self-care ability and physical and mental health. The incidence of COPD in China is relatively high. According to statistics, the incidence of COPD in people over 40 y old in China was about $8.2 \%{ }^{[1]}$ and the morbidity and mortality were increasing. COPD has the clinical characteristics of difficult to cure, long duration of the disease and ease of recurrence, which causes a waste of clinical and social resources. The annual hospitalization cost of COPD patients in China was about 2.45 billion. Elderly patients with COPD were prone to ingravescence due to complex underlying diseases and poor control of blood glucose and blood lipids. The causes of the onset and acute aggravation of COPD were not clear and it was speculated that it is related to the air pollution, infection, stress and smoking ${ }^{[2]}$. At present, the mechanism of blood glucose and blood lipids affecting the process of COPD was not clear.

*Address for correspondence

E-mail: Lilichenyi2012@163.com 
The purpose of this study was to explore the effects of dysglycemia and dyslipidemia on the severity of airflow limitation in patients with COPD, and to provide the basis for clinical treatment and prognosis of COPD. From November 2018 to November 2019, 292 patients with moderate and severe COPD were identified from a hospital. The course of disease ranged from 5 mo to $12 \mathrm{y}$, with an average of $(6.63 \pm 2.45) \mathrm{y}$. The age ranged from 43 to $86 \mathrm{y}$, with an average age of $67.3 \pm 7.8) \mathrm{y}$ old. All patients met the criteria of guidelines for primary diagnosis and treatment of chronic obstructive pulmonary disease (2018) ${ }^{[3]}$. The patients had a history of exacerbation of acute recurrence. The patients signed the informed consent form. Exclusion criteria included death or follow-up loss of contact, the patients suffered from severe lesions of the heart, kidney, liver, neuromuscular system and other important organ systems. Other diseases characterized by chronic cough, wheezing, and expectoration occurred in patients with pulmonary infection within $2 \mathrm{w}$ before enrolment, such as chronic bronchitis, bronchiectasis, cardiac insufficiency, pulmonary interstitial disease, acute and chronic pneumonia, pulmonary tuberculosis, and other respiratory diseases. A total of 292 patients were divided into 2 groups, the dysglycemia group $(n=119)$ according to the diagnostic criteria revised and published by the American Diabetes Association (ADA) in $2018^{[4]}$, and the non-dysglycemia group $(n=173)$, who were COPD patients without diabetes. In addition, the above 292 patients were diagnosed according to the diagnostic criteria of hyperlipidemia in Chinese Adult Dyslipidemia ${ }^{[5]}$ and those patients who did not take a lipid-lowering drug with triglycerides (TG) $\geq 1.7 \mathrm{mmol} / \mathrm{kg}$, or total cholesterol (TC) $\geq 5.2 \mathrm{mM}$, or high density lipoprotein cholesterol (HDL-C) $<1.0$ $\mathrm{mM}$, or low density lipoprotein cholesterol (LDL-C) $\geq 3.4 \mathrm{mM}$ within $2 \mathrm{w}$, were further divided into 2 groups, the hyperlipidemia group $(n=106)$, and the non-hyperlipidemia group $(\mathrm{n}=186)$. This study protocol was examined and approved by the Ethics Committee of the Baise People's Hospital.

Fasting venous blood $(5 \mathrm{ml})$ was collected in the morning. After serum separation, fasting blood-glucose (FBG), TC, TG, LDL-C, and HDL-C were determined on a Hitachi 7600 automatic biochemical analyser and its matching kit. The ambient temperature, humidity, and air pressure were corrected before conducting the tests to measure pulmonary function indices of all patients by the same experienced doctor using Sensormedics 6200 pulmonary function meter (Sendis Company, USA) and the average values were measured twice.
The quality control of the test refers to the pulmonary function test standard of American Thoracic Society (ATS). The measured indices including the forced expiratory volume in $1 \mathrm{~s}$ (FEV1) ${ }^{[6]}$, and FEV1/forced vital capacity (FVC), and forced expiratory volume in $1 \mathrm{sec}$ predict (FEV1 \% pred). The partial pressure of carbon dioxide in the artery $\left(\mathrm{PaCO}_{2}\right)$ and the arterial partial pressure of oxygen $\left(\mathrm{PaO}_{2}\right)$ of the patients were detected by an Omnic Blood Gas Analyzer (Roche Company, Switzerland). Dyspnea index modified by the British Medical Research Council (mMRC) was used to evaluate the degree of dyspnea. Zero score indicated that the normal walking speed of the patient is consistent with that of the normal person, and the breathing process is smooth ${ }^{[7]}$. One score indicated that the patient has shortness of breath when going uphill or brisk walking. Two score indicated that the normal walking speed of the patient is slower than that of the normal person, and there is shortness of breath in the process of walking. Three points indicated that the patients have shortness of breath after walking $100 \mathrm{~m}$ or more than $6 \mathrm{~min}$, and need to stop and have a rest. Four points indicates that the patients have obvious shortness of breath without any activity. Statistical analysis was carried out by SPSS 22.0 software (American IBM Company). The counting data were expressed in the form of $n(\%)$. The counting data were statistically analyzed by $\chi 2$ test and the grade data were statistically analysed by rank sum test. The measurement data were expressed as mean \pm standard deviation. A paired t-test was used to compare the two groups, and the difference was considered to be statistically significant $(\mathrm{p}<0.05)$. The baseline data of age, sex, weight, BMI (weight in $\mathrm{kg} /$ height in $\mathrm{m}$ ), smoking, dysglycemia and nondysglycemia, hyperlipidemia and non-hyperlipidemia group were compared (Table 1 and Table 2). And there was no significant difference in age, sex, weight, and smoking between the dysglycemia and non-dysglycemia group ( $p>0.05)$. There was no significant difference in age, sex and smoking between the hyperlipidemia and the non-hyperlipidemia group $(\mathrm{p}>0.05)$. Compared with the non-dysglycemia group, the values of FEV1, FEV1 \% pred and FEV1/FVC in the dysglycemia group were significantly higher $(\mathrm{p}<0.05)$. The values of FEV1, FEV1 \% pred and FEV1/FVC in hyperlipidemia group were significantly higher than those in the nonhyperlipidemia group $(\mathrm{p}<0.05)$. The pulmonary function of COPD patients in hyperlipidemia and dysglycemia group was significantly lower than that in the nonhyperlipidemia and non-dysglycemia group (Table 3 and Table 4). Compared to the non-dysglycemia group, 
the $\mathrm{PaCO}_{2}$ value of COPD patients in the dysglycemia group was significantly higher than that of the nondysglycemia group $(\mathrm{p}<0.05)$ and the value of $\mathrm{PaO}_{2}$ was significantly lower than that of the non-dysglycemia group ( $\mathrm{p}<0.05$ ). The $\mathrm{PaCO}_{2}$ value of COPD patients in the hyperlipidemia group was significantly higher than that of non-hyperlipidemia group $(\mathrm{p}<0.05)$, while $\mathrm{PaO}_{2}$ was significantly lower than that of non-hyperlipidemia group $(\mathrm{p}<0.05)$. The $\mathrm{mMRC}$ in dysglycemia group was significantly higher than that in non-dysglycemia group $(p<0.05)$ and mMRC in hyperlipidemia group was significantly higher than that in non-hyperlipidemia group $(\mathrm{p}<0.05)$, indicating that respiratory limitation symptoms were aggravated in COPD patients with dysglycemia and hyperlipidemia (Tables 5 and 6).

COPD has become a chronic disease with the highest incidence in China. According to the statistics of the Chinese adult lung health research, the incidence of

TABLE 1: COMPARISON OF THE BASELINE DATA IN DYSGLYCEMIA AND NON-DYSGLYCEMIA GROUPS

\begin{tabular}{lccccc}
\hline Group & Cases & Age (year) & Male $(\mathrm{n}, \%)$ & Smoking history $(\mathrm{n}, \%)$ & $\mathrm{BMl}\left(\mathrm{kg} / \mathrm{m}^{2}\right)$ \\
\hline Dysglycemia group & 119 & $69.1 \pm 5.3$ & $68(57.1 \%)$ & $74(62.2 \%)$ & $24.3 \pm 2.5$ \\
Non-dysglycemia group & 173 & $66.8 \pm 4.8$ & $93(53.8 \%)$ & $95(54.9 \%)$ & $23.7 \pm 2.8$ \\
$\mathrm{t}$ & & 1.801 & 0.724 & 1.253 & 1.878 \\
$\mathrm{p}$ & & 0.073 & 0.470 & 0.264 & 0.061 \\
\hline
\end{tabular}

TABLE 2: COMPARISON OF THE BASELINE DATA IN HYPERLIPIDEMIA AND THE NON-HYPERLIPIDEMIA GROUPS

\begin{tabular}{lccccc}
\hline Group & Cases & Age $(\mathbf{y})$ & Male $(\mathrm{n}, \%)$ & Smoking history $(\mathrm{n}, \%)$ & $\mathrm{BMI}\left(\mathrm{kg} / \mathrm{m}^{2}\right)$ \\
\hline Hyperlipidemia group & 106 & $68.5 \pm 6.7$ & $62(58.5 \%)$ & $71(67.0 \%)$ & $25.9 \pm 2.3$ \\
Non-hyperlipidemia group & 186 & $67.3 \pm 5.1$ & $99(53.2 \%)$ & $98(52.6 \%)$ & $23.4 \pm 2.6$ \\
$\mathrm{t}$ & & 1.599 & 1.766 & 1.612 & 8.232 \\
$\mathrm{p}$ & & 0.112 & 0.078 & 0.204 & 0.000 \\
\hline
\end{tabular}

TABLE 3: COMPARISON OF THE PULMONARY FUNCTION INDEX IN DYSGLYCEMIA AND NONDYSGLYCEMIA GROUPS

\begin{tabular}{lcccc}
\hline Index & Dysglycemia group $(\mathrm{n}=119)$ & Non-dysglycemia group $(\mathrm{n}=173)$ & $\mathrm{x2}$ & $\mathrm{p}$ \\
\hline FEV1 (L) & $1.32 \pm 0.08$ & $1.21 \pm 0.06$ & 12.737 & 0.000 \\
FEV1 \% pred (\%) & $54.13 \pm 2.52$ & $49.42 \pm 1.63$ & 17.967 & 0.000 \\
FEV1 /FVC (\%) & $60.25 \pm 1.74$ & $54.37 \pm 1.42$ & 30.528 & 0.000 \\
\hline
\end{tabular}

TABLE 4: COMPARISON OF THE PULMONARY FUNCTION INDEX IN HYPERLIPIDEMIA AND THE NONHYPERLIPIDEMIA GROUPS

\begin{tabular}{lcccc}
\hline Index & $\begin{array}{c}\text { Hyperlipidemia group } \\
(\mathbf{n = 1 0 6 )}\end{array}$ & $\begin{array}{c}\text { Non-hyperlipidemia group } \\
(\mathbf{n}=186)\end{array}$ & $\mathrm{x2}$ & $\mathrm{p}$ \\
\hline FEV1 (L) & $1.30 \pm 0.05$ & $1.23 \pm 0.07$ & 9.907 & 0.000 \\
FEV1 \% pred (\%) & $53.63 \pm 2.48$ & $49.88 \pm 1.71$ & 13.809 & 0.000 \\
FEV1 /FVC (\%) & $58.66 \pm 1.47$ & $55.67 \pm 1.72$ & 15.037 & 0.000 \\
\hline
\end{tabular}

TABLE 5: COMPARISON OF THE BLOOD GAS INDEX AND MMRC IN DYSGLYCEMIA AND NONDYSGLYCEMIA GROUPS

\begin{tabular}{lcccc}
\hline Index & Dysglycemia group $(\mathbf{n}=\mathbf{1 1 9})$ & Non-dysglycemia group $(\mathbf{n}=\mathbf{1 7 3})$ & $\mathrm{x} 2$ & $\mathrm{p}$ \\
\hline $\mathrm{PaCO}_{2}(\mathrm{~mm} \mathrm{Hg})$ & $58.30 \pm 4.82$ & $52.33 \pm 4.05$ & 11.085 & 0.000 \\
$\mathrm{PaO}_{2}(\mathrm{~mm} \mathrm{Hg})$ & $55.05 \pm 5.71$ & $70.21 \pm 5.82$ & 22.040 & 0.000 \\
$\mathrm{mMRC}($ score $)$ & $1.23 \pm 0.61$ & $1.09 \pm 0.52$ & 2.105 & 0.036 \\
\hline
\end{tabular}

TABLE 6: COMPARISON OF THE BLOOD GAS INDEX AND MMRC IN HYPERLIPIDEMIA AND THE NONHYPERLIPIDEMIA GROUPS

\begin{tabular}{lcccc}
\hline Index & $\begin{array}{c}\text { Hyperlipidemia group } \\
(\mathbf{n}=106)\end{array}$ & $\begin{array}{c}\text { Non-hyperlipidemia group } \\
(\mathbf{n}=186)\end{array}$ & $\mathbf{x 2}$ & $\mathrm{p}$ \\
\hline $\mathrm{PaCO}_{2}(\mathrm{~mm} \mathrm{Hg})$ & $56.03 \pm 5.22$ & $51.93 \pm 4.35$ & 6.845 & 0.000 \\
$\mathrm{PaO}_{2}(\mathrm{~mm} \mathrm{Hg})$ & $54.65 \pm 4.03$ & $69.31 \pm 4.62$ & 27.282 & 0.000 \\
mMRC $^{\text {score })}$ & $1.25 \pm 0.61$ & $1.10 \pm 0.57$ & 2.108 & 0.035 \\
\hline
\end{tabular}


COPD in adults aged 20 and over was $8.6 \%$ in 2018, with nearly 100 million patients. Researchers claim more the urbanization, more the air pollution thus severity of air flow limitation which must be controlled. The incidence of COPD over $40 \mathrm{y}$ old increased to $13.7 \%{ }^{[8]}$. At present, COPD was the third most common chronic disease after hypertension and diabetes. The World Health Organization (WHO) found that COPD was the disease with the heaviest economic burden in China. In addition, the mortality rate of COPD was high. In 2013, the number of COPD deaths in China was about 911000 , accounting for more than $1 / 3$ of the global COPD mortality in the same year, and the mortality rate was expected to increase ${ }^{[9]}$. The main symptoms of COPD were pulmonary inflammation, airflow limitation, emphysema pulmonum, and the weakening or loss of pulmonary ciliary motor function. At present, there was no effective prevention and treatment of COPD in the clinic. And the pathogenesis and progressive factors of COPD were not completely clear. It was reported that COPD was related to the long-term stimulation of air harmful particles to the lungs and abnormal inflammatory response ${ }^{[10]}$. COPD mostly occurs in middle-aged and elderly people, and the incidence was positively correlated with age, which is speculated to be related to the deterioration of immune function, and poor control of blood glucose and blood lipids in the elderly. In this study, the clinical indices of COPD patients with dyslipidemia and dysglycemia were analysed. One-way ANOVA was used to analyse the relationship between pulmonary function and blood gas index, and mMRC in COPD patients complicated with type 2 diabetes and hyperlipidemia. This study explored whether diabetes and dyslipidemia were the risk factors of airflow limitation in COPD, and then to provide a theoretical basis for risk assessment, prevention, and treatment of COPD. In this study, it was found that the pulmonary function indexes FEV1, FEV1 \% pred, and FEV1/FVC of COPD patients complicated with diabetes were significantly higher than those of nondiabetic patients, indicating that the pulmonary function of COPD patients was affected by the diabetes, which would aggravate the degree of airflow limitation in COPD patients. In addition, the blood gas index $\mathrm{PaCO}_{2}$ increased and $\mathrm{PaO}_{2}$ decreased in COPD patients with diabetes, indicating that diabetes has a great influence on pulmonary hemodynamic and worsens the signs and prognosis of COPD. The routine clinical evaluation method of COPD symptoms was mMRC grade or CAT score. The mMRC score of COPD patients complicated with diabetes increased significantly, indicating that the decrease of pulmonary function in patients with diabetes affected their respiratory smoothness and respiratory efficiency. Diabetes was one of the risk factors for COPD patients. Diabetes was a systemic metabolic disorder, which endangers all organs of the body, and it was easy to be complicated with serious diseases of various organs, such as hypertension, diabetic nephropathy, cardiovascular disease, cerebrovascular disease, diabetic neuropathy. Recent studies have shown that the lung was also a target organ threatened by diabetes. The histopathological study of the lungs of the diabetic showed that a lot of elastin and collagen were secreted by the extracellular matrix of alveoli cell $^{[11]}$, and the basal lamina of alveolar epithelial cells and alveolar endothelial cell thickened obviously ${ }^{[12,13]}$. The ultrastructure of alveolar epithelial cells was also abnormal ${ }^{[14]}$. These pathological changes of diabetes in the lungs may be the pathological structural basis of the airflow limitation and the deterioration of blood gas indexes in COPD patients complicated with diabetes. In view of the fact that COPD was a systemic inflammatory disease, the levels of white blood cells, C-reactive protein (CRP), tumour necrosis factor- $\alpha$, and fibrinogen in COPD patients were significantly increased $^{[15]}$. The levels of inflammatory factors such as tumour necrosis factor- $\alpha$, IL- 6 , and CRP in patients with diabetes were also abnormally increased, and the correlation between diabetes and COPD can be explained at the molecular level ${ }^{[16]}$. The effect of dyslipidemia on airflow limitation in patients with COPD was also studied. The results showed that the pulmonary function indexes FEV1, FEV1 \% pred, and FEV1/FVC in hyperlipidemic COPD patients was significantly higher than those in non-hyperlipidemic patients, indicating that pulmonary function decreased and airflow limitation increased in hyperlipidemic COPD patients. Hyperlipidemia was a risk factor for COPD patients. The blood gas index $\mathrm{PaCO}_{2}$ increased and $\mathrm{PaO}_{2}$ decreased in COPD patients complicated with hyperlipidemia, indicating that hyperlipidemia significantly decreased pulmonary hemodynamics and worsened the blood oxygen level of COPD and prognosis. The mMRC score of hyperlipidemia patients with COPD increased significantly, indicating that the dyspnea degree of COPD patients with hyperlipidemia was aggravated, which affected the body function and respiratory efficiency. Hyperlipidemia was also one of the risk factors for patients with COPD. The specific mechanism of dysglycemia and dyslipidemia aggravating the prognosis of COPD and its correlation with the pulmonary function was not clear, and 
further research is needed. To sum up, diabetes and hyperlipidemia were risk factors of COPD, which can reduce pulmonary function, deepen airflow limitation, significantly reduce pulmonary hemodynamics and aggravate dyspnea. This study has important guiding significance for clinical prevention and treatment of COPD.

\section{Conflict of interest:}

All authors report no conflicts of interest in this work.

\section{REFERENCES}

1. Halbert RJ, Isonaka S, George D, Iqbal A. Interpreting COPD prevalence estimates what is the true burden of disease? Chest 2003;1:1684-92.

2. He S, Chen D, Hu M, Zhang L, Liu C, Traini D, et al. Bronchial epithelial cell extracellular vesicles ameliorate epithelialmesenchymal transition in COPD pathogenesis by alleviating M2 macrophage polarization. Nanomedicine 2019:259-71.

3. Chinese Medical Association, Journal of Chinese Medical Association, Chinese society of general practice, et al. Guidelines for primary diagnosis and treatment of chronic obstructive pulmonary disease. Chin J Gen Pract 2018;17:85670 .

4. American Diabetes Association. Cardiovascular Disease and Risk Management: Standards of Medical Care in Diabetes-2018. Diabetes Care 2018;41:S86-S104.

5. Chinese Joint Committee for revision of guidelines for Prevention and treatment of Adult Dyslipidemia. Guidelines for the Prevention and Control of Hyperlipidemia in Chinese Adults: 2016 revised edition. Chin Circulation J 2016,;31(10):937.

6. Miller MR, Hankinson J, Brusasco V. Standardisation of spirometry. Eur Respir J 2005;26:319-38.

7. American Thoracic Society. ATS statement: guidelines for the six-min walk test. Am J Respir Crit Care Med 2002;166:111-7.
8. Zhu HL. Current situation and progress of chronic disease management in elderly patients with chronic obstructive pulmonary disease. Geriatr Health Care 2018;24:219-22.

9. Mei L, Wu S, Wang D, Li H, Zhang H, Wang M. Epidemiology of Dementia in Elderly Chronic Obstructive Pulmonary Disease Patients Living in China's Northwestern High-Elevation Area. Med Sci Monit 2018;9:76-9.

10. Han F, Yang X, Xu D, Wang Q, Xu D. Association between outdoor PM 2.5 and prevalence of COPD: a systematic review and meta-analysis. Postgrad Med J 2019;95:612-8.

11. Sahebjami H, Denholm D. Lung mechanics and connective tissue proteins in diabetic Bio-Breeding Worcester Wistar rats. J Appl Physiol 1987;62:1430-5.

12. Popov D, Simionescu M. Alterations of lung structure in experimental di- abetes, and diabetes associated with hyperlipidaemia in hamsters. Eur Respir 1997;10:1850-8.

13. Weynand B, Jonckheere A, Frans A, Rahier J. Diabetes mellitus induces a thickening of the pulmonary basal lamina. Respiration 1999;66:14-9.

14. Sugahara K, Morioka T. Studies of the lungs in diabetes mellitus. Respir Circ 1983;31:1287-94.

15. Gan WQ, Man SF, Senthilselvan A, Sin DD. Association between chronic obstructive pulmonary disease and systemic inflammation: a systematic review and a meta-analysis. Thorax 2004;59:574-80.

16. Hu FB, Meigs JB, Li TY, Rifai N, Manson JE. Inflammatory Markers and Risk of Developing Type 2 Diabetes in Women. Diabetes 2004;53:693-700.4.

This is an open access article distributed under the terms of the Creative Commons Attribution-NonCommercial-ShareAlike 3.0 License, which allows others to remix, tweak, and build upon the work non-commercially, as long as the author is credited and the new creations are licensed under the identical terms

This article was originally published in a special issue, "Biomedical Research in Clinical and Preclinical Pharmaceutics" Indian J Pharm Sci 2020:82(3)Spl issue7;1-5 